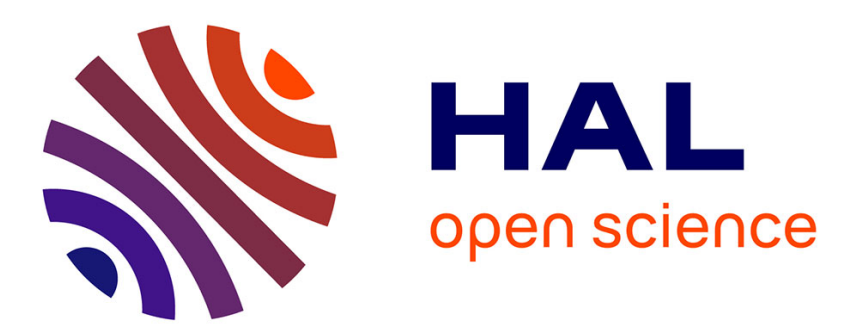

\title{
On output regulation in state-constrained systems: An application to polyhedral case
}

Aneel Tanwani, Bernard Brogliato, Christophe Prieur

\section{To cite this version:}

Aneel Tanwani, Bernard Brogliato, Christophe Prieur. On output regulation in state-constrained systems: An application to polyhedral case. IFAC WC 2014 - 19th IFAC World Congress, Aug 2014, Le Cap, South Africa. 10.3182/20140824-6-ZA-1003.01477 . hal-00997266v2

\section{HAL Id: hal-00997266 \\ https://hal.science/hal-00997266v2}

Submitted on 21 Feb 2015

HAL is a multi-disciplinary open access archive for the deposit and dissemination of scientific research documents, whether they are published or not. The documents may come from teaching and research institutions in France or abroad, or from public or private research centers.
L'archive ouverte pluridisciplinaire HAL, est destinée au dépôt et à la diffusion de documents scientifiques de niveau recherche, publiés ou non, émanant des établissements d'enseignement et de recherche français ou étrangers, des laboratoires publics ou privés. 


\title{
On output regulation in state-constrained systems: An application to polyhedral case
}

\author{
Aneel Tanwani* Bernard Brogliato** Christophe Prieur * \\ * Gipsa-lab, Department of Automatic Control, 11 Rue des \\ Mathématiques, BP 46, 38402 Saint Martin d'Hères, France. \\ Email: $\{$ aneel.tanwani, christophe.prieur\}@gipsa-lab.fr \\ ** Team BipOp, INRIA Rhône-Alpes, 655 Avenue de l'Europe, \\ 38334 Montbonnot Cedex, France. \\ Email: bernard.brogliato@inria.fr
}

\begin{abstract}
This paper deals with the problem of output regulation using the state feedback control laws for a class of nonsmooth dynamical systems where the state is constrained to evolve within some convex set. The formalism of differential inclusions (DIs) is used to describe the system dynamics and the derivation of the state feedback law is based on the internal model principle. We study two types of control laws: firstly, a static control is designed assuming that the entire states of the plant and the exosystem are available for feedback; In the second case, only the error to be regulated is available for feedback and a dynamic compensator is designed. The analyses are based on using the properties of the normal cones associated with convex sets to study the well-posedness (existence and uniqueness of solutions) and the stability of the closed-loop system. As an application, we design a discontinuous controller which guarantees the viability of a predefined polyhedral subset of the state space using the formulation of linear complementarity systems.
\end{abstract}

\section{INTRODUCTION}

Output regulation is one of the classical system theoretic problems which relates to designing control laws for asymptotically tracking a reference trajectory or rejecting disturbances. This paper addresses the problem of output regulation for a class of linear systems where the state trajectory is contained within a time-varying set. Such motions are modeled by differential inclusions where the multivalued part is due to the subdifferential of the indicator function (which is equivalent to the normal cone for convex sets) of the set containing the state trajectory, see (4) below for exact formulation.

The approach adopted in this paper is based on the pioneering work of Francis [1977] who proposed simple algebraic criteria for output regulation in multivariable linear time-invariant (LTI) systems. In his work, it is assumed that the plant under consideration is driven by the output of an LTI exosystem that models the dynamics of the reference trajectories and/or disturbances. Intuitively speaking, the proposed control input that achieves the output regulation comprises a feedback component to make the closed-loop dynamics stable and an additional openloop component that shapes the steady state of the plant. The derivation of the open-loop component of the control input requires the exact knowledge of the exosystem dynamics, and hence the approach is termed as internal model principle. A large amount of work has followed on the problem of output regulation using internal model principle in more general contexts, see Byrnes and Isidori

* This work has been partially supported by the LabEx PERSYVAL-Lab (ANR-11-LABX-0025-01).
[1990, 2000], Serrani et al. [2001] and the references therein for the results related to nonlinear systems and robustness with respect to uncertainty in system parameters.

More recently, the problem of output regulation has been studied in the context of nonsmooth systems (where the right-hand side of the differential equations describing the dynamics is either discontinuous or set-valued) as well using several approaches. In the work of Benjamin Biemond et al. [2013], Forni et al. [2013], Morarescu and Brogliato [2010], Lyapunov based methods are proposed for tracking reference trajectories in systems subjected to impacts that result in discontinuous state trajectories. Inversionbased methods are proposed for generating desired output trajectories for switched systems by Tanwani and Liberzon [2010], Vu and Liberzon [2008]. The discontinuous variants of internal model principle have also been developed to study the problem of output regulation in switched systems with parameter uncertainties by Galeani et al. [2012], and systems with state resets by Marconi and Teel [2013]. One also finds the application of internal model principle in the formation control and output synchronization problems in networked systems due to Wieland et al. [2011], De Persis and Jayawardhana [2012].

The approach adopted in this paper generalizes the internal model principle for systems that are constrained to evolve within some predefined time-varying convex set. For simplicity, we will consider the case where the variation of the set-valued mapping is absolutely continuous (with respect to the Hausdorff metric that measures the distance between sets at different time instants). The states of the exosystem and the plant are assumed to evolve within the same sets and we derive sufficient conditions under which 
there exists a control input that achieves output regulation while maintaining state constraints. In addition to the classical regulator synthesis equations, additional conditions are needed in our work to generate a dissipative relation between the multivalued part and the output regulation error. These additional conditional conditions also guarantee that the closed-loop system is well-posed, that is, it admits a unique solution which is an important consideration for designing controllers for such class of systems. We study two cases for control synthesis depending on how much information is available to the controller. In the first case, it is assumed that the entire states of the plant and the exosystem are available and thus, a static controller is designed to achieve output regulation. In the second case, it is assumed that only the regulation error (which needs to converge to zero) is available and in that case a dynamic compensator is designed. The analysis is based on making the closed-loop system passive and conceptually similar ideas could be found for state estimation problems in Brogliato and Heemels [2009], Heemels et al. [2011]. As an application, we consider reference signals generated by linear complementarity systems (LCS), and design control input for an LTI system that not only achieves the output regulation but also forces the state-trajectory to evolve within a predefined time-varying polyhedron in the state space.

The remainder of the paper is organized as follows: In Section 2, we describe the system class and some fundamental results along with the formal definition of the problem addressed in this paper. Section 3 deals with the design of static state feedback control when using the full state measurement. In case of partial measurements, the dynamic compensator is designed in Section 4. Finally, these results are applied to derive a discontinuous viability control to maintain state constraints in Section 5 .

\section{PRELIMINARIES}

Before stating the system class and the problem formulation, let us introduce a few preliminaries that lay the foundation of results in this paper. Consider a set-valued map $\mathcal{S}:[0, \infty) \rightrightarrows \mathbb{R}^{n}$ where $\mathcal{S}(t), \forall t \geq 0$, is nonempty, closed and convex. We are interested in modeling the motion of a state variable $x$ governed by an ordinary differential equation, say $\dot{x}(t)=f(t, x(t))$ under the constraint that $x(t) \in \mathcal{S}(t), \forall t \geq 0$. Intuitively speaking, as long as $x(t)$ is in the interior of the set, we need not do anything to the equation $\dot{x}(t)=f(t, x(t)$ ) (for at least a small period of time) to satisfy the constraint, until the state $x(t)$ hits the boundary of the set $\mathcal{S}(t)$. At this moment, if the vector field $f(t, x(t))$ is pointed outside of the set $\mathcal{S}(t)$, then any component of this vector field in the direction normal to $\mathcal{S}(t)$ at $x(t)$ must be annihilated to maintain the motion of $x$ within the constraint set. With this motivation, we introduce the following differential inclusion and the dynamical systems treated in this paper, are basically of this form.

$$
\dot{x}(t) \in f(t, x(t))-\mathcal{N}(\mathcal{S}(t) ; x(t)), \quad \text { Lebesgue a.e. } t \geq 0
$$

with the initial condition $x(0)=\mathcal{S}(0)$. Here we used the notation $\mathcal{N}(S ; x):=\left\{\lambda \in \mathbb{R}^{n}:\langle\lambda, z-x\rangle \leq 0, \forall z \in S\right\}$ to denote the normal cone to a convex set $S$ at a point $x \in S$. We adopt the conventions that $\mathcal{N}(S, x)=\emptyset$ when $x \notin S$. It follows from the definition that $\mathcal{N}(S, x)=\{0\}$ when $x$ is in the interior of $S$ and that for nonempty, closed and convex sets $S$, the operator $\mathcal{N}(S, \cdot)$ is monotone ${ }^{1}$. The differential inclusion in (1) is termed as a perturbed sweeping process, and was introduced in Moreau [1977].

Remark 1. It must be noted that equation (1) doesn't model the motion of constrained mechanical systems, such as a moving ball subjected to impacts. The primary difference between the two cases is that (1) gives a possible solution that stays in the set $\mathcal{S}(t)$ whereas in mechanical systems, we are looking to model a specific behavior at the impacts which must be specified through an impact law. Nonetheless, mechanical systems with impacts have been modeled using formalisms closely related to (1), where the set $\mathcal{S}(\cdot)$ is actually state-dependent and not necessarily just time-dependent, see [Brogliato, 1999, Chapter 5].

Let us now address the existence and uniqueness of solutions for the inclusion given in (1). For that, let us introduce the following hypothesis for the set-valued map $\mathcal{S}:[0, \infty) \rightrightarrows \mathbb{R}^{n}:$

(H1) For each $t \in[0, \infty), \mathcal{S}(t)$ is a non-empty, closed and convex set.

(H2) The variation in $\mathcal{S}(\cdot)$ is absolutely continuous; that is, there exists a locally absolutely continuous function $\nu(\cdot)$ such that for any $y \in \mathbb{R}^{n}$, and $s, t \geq 0$

$$
|d(y, \mathcal{S}(t))-d(y, \mathcal{S}(s))| \leq|\nu(t)-\nu(s)|
$$

where $d(y, S):=\inf _{z \in S}|y-z|$.

These hypotheses are used to guarantee the existence of solutions in the following result that appeared in Edmond and Thibault [2005].

Theorem 2. (Existence and uniqueness). Consider system (1) over some interval $I \subseteq\left[t_{0}, \infty\right)$ where the set-valued map $\mathcal{S}(\cdot)$ satisfies the hypotheses (H1) and (H2). Assume that $f(t, x)$ satisfies the following assumptions:

- for each $x \in \mathbb{R}^{n}, f(\cdot, x)$ is a Lebesgue-measurable function,

- for each $t \in I, f(t, \cdot)$ is Lipschitz continuous, and

- there exists a locally integrable function $\alpha: I \rightarrow \mathbb{R}$ such that $|f(t, x)| \leq \alpha(t)(1+|x|)$ for all $x \in \cup_{s \in I} \mathcal{S}(s)$.

Then, for each $x_{0} \in \mathcal{S}(0)$, there exists a unique locally absolutely continuous solution $x(\cdot)$ that satisfies (1), and that $x(t) \in \mathcal{S}(t)$ for all $t \in I$.

Before proceeding towards the problem formulation, let us recall the following basic result from convex analysis which will be used later in the well-posedness analysis of the closed-loop system.

Lemma 3. (Chain Rule). Consider a nonempty, closed, convex set $S \subseteq \mathbb{R}^{d_{s}}$, and a linear map $H: \mathbb{R}^{n} \rightarrow \mathbb{R}^{d_{s}}$. Let $S^{\prime}:=H^{-1}(S)$, and assume that $H$ satisfies the following constraint qualification:

$$
\forall z \in S^{\prime} \text { and } \lambda \in \mathcal{N}(S ; H z), H^{\top} \lambda=0 \Rightarrow \lambda=0 .
$$

Then, for each $z \in \mathbb{R}^{n}$, and $\bar{v}=H z$, it holds that

$$
\mathcal{N}\left(S^{\prime} ; z\right):=\left\{H^{\top} \lambda \mid \lambda \in \mathcal{N}(S ; \bar{v})\right\}=H^{\top} \mathcal{N}(S ; H z) .
$$

Remark 4. If $H$ has full row rank, that is $\operatorname{rank} H=d_{s}$, then the constraint qualification (2) holds automatically.

\footnotetext{
1 A multivalued map $\phi: \mathbb{R}^{n} \rightrightarrows \mathbb{R}^{m}$ is called monotone, if for each $x_{1}, x_{2}$ in the domain of $\phi$, it holds that $\left\langle y_{2}-y_{1}, x_{2}-x_{1}\right\rangle \geq 0$ for each $y_{i} \in \phi\left(x_{i}\right), i=1,2$.
} 
However, (2) does not imply that $H$ has full row rank. An equivalent way of expressing (2) is

$$
\operatorname{ker}\left(H^{\top}\right) \cap \mathcal{N}(S ; H z)=\{0\}, \quad \forall z \in H^{-1}(S),
$$

where $\operatorname{ker}(\cdot)$ denotes the kernel/ null space. For convex sets, (2) also holds if the set $S$ cannot be separated from the range of matrix $H$, see [Rockafellar and Wets, 1998, Theorem 10.6].

\subsection{Problem Formulation}

Consider a set-valued mapping $\mathcal{S}:[0, \infty) \rightrightarrows \mathbb{R}^{d_{s}}$ which satisfies (H1) and (H2). The class of systems we consider in this paper is defined as follows:

$$
\begin{gathered}
\dot{x}(t)=A x(t)+B u(t)+F x_{r}(t)+G \lambda \\
\lambda \in-\mathcal{N}(\mathcal{S}(t) ; H x(t))
\end{gathered}
$$

where $x_{r}:[0, \infty) \rightarrow \mathbb{R}^{d_{r}}$ is the reference signal that is generated from the following equations:

$$
\begin{gathered}
\dot{x}_{r}(t)=A_{r} x_{r}(t)+G_{r} \lambda_{r} \\
\lambda_{r} \in-\mathcal{N}\left(\mathcal{S}(t) ; H_{r} x_{r}(t)\right) .
\end{gathered}
$$

The output regulation variable $w$ is defined as:

$$
w(t)=C x(t)+D x_{r}(t) .
$$

We say that the output regulation is achieved if there exists a control input $u$ such that the following properties are satisfied:

- Well-posedness: For each initial condition $x(0)$ satisfying $H x(0) \in \mathcal{S}(0)$, there exists a unique locally absolutely continuous solution $x(\cdot)$ such that $H x(t) \in \mathcal{S}(t)$, $\forall t \geq 0$.

- Regulation: It holds that $\lim _{t \rightarrow \infty} w(t)=0$.

- Closed-loop stability: The plant and controller dynamics have a globally asymptotically stable equilibrium at the origin when $x_{r} \equiv 0$.

In addition to the hypotheses (H1) and (H2) introduced earlier, the following statements are always assumed to hold, and are considered a part of problem statement for the results in this paper:

(H3) The matrix $H$ introduced in (4b) satisfies the constraint qualification (2) for each $\mathcal{S}(t), t \geq 0$.

(H4) System (5) is initialized with $H_{r} x_{r}(0) \in \mathcal{S}(0)$, and there exists at least one locally absolutely continuous solution $x_{r}(\cdot)$ such that $H_{r} x_{r}(t) \in \mathcal{S}(t)$, for each $t \geq 0$.

We consider two cases: first, it is assumed that the states $x$ and $x_{r}$ are available to the controller and hence a static state feedback law is designed; secondly, it is assumed that only the regulation error $w$ is available for feedback and in that case a dynamic compensator is designed.

\section{STATIC STATE FEEDBACK CONTROL}

In this section, we assume that all the states of the plant (4) and (5) are available for feedback and thus a control input with static state feedback can be designed which achieves the stability and regulation. In the formulation of our results, the following terminology is used: A triplet of matrices $(A, G, H)$ is called strictly passive if there exist symmetric positive definite matrices $P, Q$ such that

$$
\begin{gathered}
A^{\top} P+P A=-Q \\
P G=H^{\top}
\end{gathered}
$$

Theorem 5. Suppose that a matrix $K$ renders the triplet $(A+B K, G, H)$ strictly passive, and that there exist matrices $\Pi \in \mathbb{R}^{n \times d_{r}}$ and $M \in \mathbb{R}^{d_{u} \times d_{r}}$ such that

$$
\begin{gathered}
\Pi A_{r}=A \Pi+B M+F \text { and } C \Pi+D=0 \\
\Pi G_{r}=G \text { and } H \Pi=H_{r} .
\end{gathered}
$$

Then the output regulation problem is solvable with the following static feedback control law:

$$
u(t)=K x(t)+(M-K \Pi) x_{r}(t) .
$$

Remark 6. In the work of Francis [1977], the control law (9) was proposed to solve the output regulation problem in LTI systems, where $\Pi$ and $M$ were obtained as solution of (8a) only, and $K$ is any matrix that makes $(A+B K)$ Hurwitz. The strict passivity requirement, and additional conditions on the matrix $\Pi$ in $(8 \mathrm{~b})$ are required in the wellposedness and stability analyses for the class of systems considered in this paper.

Proof. a) Well-posedness: With control input (9), the closed-loop system is written as

$$
\begin{gathered}
\dot{x}(t)=(A+B K) x(t)+(F+B M-B K \Pi) x_{r}(t)+G \lambda \\
\lambda \in-\mathcal{N}(\mathcal{S}(t) ; H x(t)) .
\end{gathered}
$$

Let $P$ be the matrix such that $P G=H^{\top}$ (which follows due to passivity of $(A+B K, G, H))$ and $R$ be the symmetric positive definite matrix such that $R^{2}=P$. Introduce the coordinate transformation $z=R x$, then

$$
\begin{aligned}
& \dot{z}(t)= R(A+B K) R^{-1} z(t) \\
& \quad+R(F+B M-B K \Pi) x_{r}(t)+R G \lambda \\
& \lambda \in-\mathcal{N}\left(\mathcal{S}(t) ; H R^{-1} z(t)\right)
\end{aligned}
$$

By assumption, $R G=R^{-1} H^{\top}$, and from Lemma 3 , $R G \lambda=R^{-1} H^{\top} \lambda \in-\mathcal{N}\left(\mathcal{S}^{\prime}(t) ; z(t)\right)$ where $\mathcal{S}^{\prime}(t):=\{z \in$ $\left.\mathbb{R}^{n} \mid H R^{-1} z \in \mathcal{S}(t)\right\}$. Thus, system (10) is in the standard form (1), and the existence and uniqueness of solutions now follows from Theorem 2.

b) Regulation: Let $\Pi$ be the matrix that satisfies (8) and introduce the variable $e=x-\Pi x_{r}$. The output regulation is achieved if we can show that $\lim _{t \rightarrow \infty} e(t)=0$, since

$$
\begin{aligned}
w(t) & =C x(t)+D x_{r}(t) \\
& =C x(t)-C \Pi x_{r}(t)=C e(t) .
\end{aligned}
$$

To show that $e \rightarrow 0$, we observe that

$$
\begin{aligned}
\dot{e}(t)= & (A+B K) x(t)+(F+B M-B K \Pi) x_{r}(t) \\
& \quad+G \lambda-\Pi A_{r} x_{r}(t)-\Pi G_{r} \lambda_{r} \\
= & (A+B K) x(t)-(\underbrace{\Pi A_{r}-F-B M}_{=A \Pi}+B K \Pi) x_{r}(t) \\
& \quad+G \lambda-\Pi G_{r} \lambda_{r} \\
= & (A+B K) x(t)-(A+B K) \Pi x_{r}(t)+G \lambda-\Pi G_{r} \lambda_{r} \\
= & (A+B K) e(t)+G\left(\lambda-\lambda_{r}\right) .
\end{aligned}
$$

Now introduce the Lyapunov function $V(e)=e^{\top} P e$, so that the following holds for almost all $t \geq 0$ :

$$
\begin{aligned}
\dot{V}(e(t))= & e(t)^{\top}\left((A+B K)^{\top} P+P(A+B K)\right) e(t) \\
& +2 e(t)^{\top} P G\left(\lambda-\lambda_{r}\right) \\
= & -e(t)^{\top} Q e(t)+2 e(t)^{\top} H^{\top}\left(\lambda-\lambda_{r}\right) \\
= & -e(t)^{\top} Q e(t)+\left\langle\lambda-\lambda_{r}, H x(t)-H \Pi x_{r}(t)\right\rangle \\
= & -e(t)^{\top} Q e(t)+\left\langle\lambda-\lambda_{r}, H x(t)-H_{r} x_{r}(t)\right\rangle .
\end{aligned}
$$

For every feasible solution of (4) and (5), it holds that $\left\langle\lambda, H x(t)-H_{r} x_{r}(t)\right\rangle \leq 0$ and $\left\langle\lambda_{r}, H x(t)-H_{r} x_{r}(t)\right\rangle \geq 0$. 
It thus follows that $\dot{V}(e(t))<0$ for all $e(t) \neq 0$, and thus $e(t) \rightarrow 0$.

Closed-loop stabilization: Following the same calculations as above, it is seen that the derivative of $V$ along the dynamics of (4) with $u$ in (9) satisfies

$\dot{V}(x(t)) \leq-x(t)^{\top} Q x(t)+x(t)^{\top} P(F+B(M-K \Pi)) x_{r}(t)$

from where it follows that $x(t) \rightarrow 0$ when $x_{r} \equiv 0$.

Remark 7. The reference system may not have a unique solution under $(\mathbf{H} 4)$, but the closed-loop system always has a unique solution for each initial condition satisfying $H x(0) \in \mathcal{S}(0)$ and a given $x_{r}$ obtained from (5).

\section{DYNAMIC COMPENSATOR}

In this section, it will no longer be assumed that the states $x$ and $x_{r}$ are available for feedback, but only the regulation error $w$ is available to the controller. Our approach is based on the certainty equivalence principle where we first design an estimator for the state variables $x$ and $x_{r}$ and then define the control law as a function of these estimates.

Towards this end, the estimator we propose is defined as follows:

$$
\begin{aligned}
&\left(\begin{array}{c}
\dot{\hat{x}} \\
\hat{\hat{x}}_{r}
\end{array}\right)=\left[\left(\begin{array}{cc}
A & F \\
0 & A_{r}
\end{array}\right)\right.\left.-\left(\begin{array}{c}
L_{0} \\
L_{1}
\end{array}\right)\left(\begin{array}{ll}
C & D
\end{array}\right)\right]\left(\begin{array}{c}
\hat{x} \\
\hat{x}_{r}
\end{array}\right)+\left(\begin{array}{c}
L_{0} \\
L_{1}
\end{array}\right) w \\
&+\left(\begin{array}{c}
B \\
0
\end{array}\right) u+\left(\begin{array}{cc}
G & 0 \\
0 & G_{r}
\end{array}\right)\left(\begin{array}{c}
\hat{\lambda} \\
\hat{\lambda}_{r}
\end{array}\right) \\
&\left(\begin{array}{c}
\hat{\lambda} \\
\hat{\lambda}_{r}
\end{array}\right) \in-\mathcal{N}\left(\mathcal{S}(t) \times \mathcal{S}(t) ;\left(\begin{array}{cc}
H & 0 \\
0 & H_{r}
\end{array}\right)\left(\begin{array}{c}
\hat{x} \\
\hat{x}_{r}
\end{array}\right)\right),
\end{aligned}
$$

where the gain matrix $L:=\left(\begin{array}{l}L_{0} \\ L_{1}\end{array}\right)$ will be designed in the sequel. For brevity, let us introduce the following notation:

$$
\begin{gathered}
\bar{A}:=\left(\begin{array}{cc}
A & F \\
0 & A_{r}
\end{array}\right), \quad \bar{C}:=\left(\begin{array}{ll}
C & D
\end{array}\right), \\
\bar{G}:=\left(\begin{array}{cc}
G & 0 \\
0 & G_{r}
\end{array}\right), \quad \bar{H}:=\left(\begin{array}{cc}
H & 0 \\
0 & H_{r}
\end{array}\right) .
\end{gathered}
$$

Theorem 8. Suppose that there exist a feedback matrix $K$ and an injection matrix $L$ that render the triplets $(A+B K, G, H)$ and $(\bar{A}-L \bar{C}, \bar{G}, \bar{H})$ strictly passive, respectively. If there exist matrices $\Pi \in \mathbb{R}^{n \times d_{r}}$ and $M \in \mathbb{R}^{d_{u} \times d_{r}}$ that satisfy (8), then the output regulation problem is solved by letting

$$
u(t)=K \hat{x}(t)+(M-K \Pi) \hat{x}_{r}(t) .
$$

Proof. Introduce the variable $\tilde{x}:=x-\hat{x}$, then the closedloop dynamics are similar to

$$
\begin{aligned}
\left(\begin{array}{c}
\dot{x} \\
\dot{\tilde{x}} \\
\dot{\hat{x}}_{r}
\end{array}\right)= & \underbrace{\left(\begin{array}{ccc}
A+B K & -B K & B(M-K \Pi) \\
0 & A-L_{0} C & -F+L_{0} D \\
0 & L_{1} C & A_{r}-L_{1} D
\end{array}\right)}_{:=A_{c l}}\left(\begin{array}{c}
x \\
\tilde{x} \\
\hat{x}_{r}
\end{array}\right) \\
& +\underbrace{\left(\begin{array}{c}
F-L_{0} D \\
L_{1} D
\end{array}\right)}_{:=F_{c l}} x_{r}+\underbrace{\left(\begin{array}{ccc}
G & 0 & 0 \\
G & -G & 0 \\
0 & 0 & G_{r}
\end{array}\right)}_{:=G_{c l}}\left(\begin{array}{c}
\lambda \\
\hat{\lambda} \\
\hat{\lambda}_{r}
\end{array}\right)
\end{aligned}
$$

$\left(\begin{array}{c}\lambda \\ \hat{\lambda} \\ \hat{\lambda}_{r}\end{array}\right) \in-\mathcal{N}(\mathcal{S}(t) \times \mathcal{S}(t) \times \mathcal{S}(t), \underbrace{\left(\begin{array}{ccc}H & 0 & 0 \\ H & -H & 0 \\ 0 & 0 & H_{r}\end{array}\right)}_{:=H_{c l}}\left(\begin{array}{c}x \\ \tilde{x} \\ \hat{x}_{r}\end{array}\right))$.

Well-posedness: To show that the closed-loop system admits a unique solution, we follow the same procedure as in the proof of Theorem 5. That is, we find a symmetric positive definite matrix $P_{c l}$ such that $P_{c l} G_{c l}=H_{c l}^{\top}$. We can then take the positive square root of this matrix, denoted by $R_{c l}$, and define a coordinate transformation that brings the system in the form of (1).

To find the matrix $P_{c l}$, it is noted that, by assumption, the triplets $(A+B K, G, H)$ and $(\bar{A}-L \bar{C}, \bar{G}, \bar{H})$ are passive, so there exist symmetric positive definite matrices $P, Q, \bar{P}, \bar{Q}$ such that

$$
\begin{aligned}
& (A+B K)^{\top} P+P(A+B K)=-Q, \quad P G=H^{\top}, \\
& (\bar{A}-L \bar{C})^{\top} \bar{P}+\bar{P}(\bar{A}-L \bar{C})=-\bar{Q}, \quad \overline{P G}=\bar{H}^{\top} .
\end{aligned}
$$

Now, partition $\bar{P}$ as $\bar{P}:=\left(\begin{array}{ll}\bar{P}_{11} & \bar{P}_{12} \\ \bar{P}_{12} & \bar{P}_{22}\end{array}\right)$, then $\bar{P}_{22}$ is a symmetric positive definite matrix, and $\bar{P}_{22} G_{r}=H_{r}^{\top}$. Thus, by letting

$$
P_{c l}:=\left[\begin{array}{ccc}
2 P & -P & 0 \\
-P & P & 0 \\
0 & 0 & \bar{P}_{22}
\end{array}\right]
$$

it is easily checked that $P_{c l}$ is symmetric, positive definite, and $P_{c l} G_{c l}=H_{c l}^{\top}$.

Regulation: By letting $W:=(-K(M-K \Pi))$, it is seen that

$$
A_{c l}=\left(\begin{array}{cc}
A+B K & B W \\
0 & \bar{A}-L \bar{C}
\end{array}\right)
$$

Define the regulation error as follows:

$$
e:=\left(\begin{array}{c}
x-\Pi x_{r} \\
\tilde{x} \\
\hat{x}_{r}-x_{r}
\end{array}\right)=:\left(\begin{array}{c}
e_{x} \\
\tilde{x} \\
e_{r}
\end{array}\right)=:\left(\begin{array}{c}
e_{x} \\
e_{\xi}
\end{array}\right)
$$

then it can be shown using the equations in (8) that

$$
\dot{e}(t)=\left(\begin{array}{cc}
A+B K & B W \\
0 & \bar{A}-L \bar{C}
\end{array}\right) e(t)+\left(\begin{array}{ccc}
G & 0 & 0 \\
0 & G & 0 \\
0 & 0 & G_{r}
\end{array}\right)\left(\begin{array}{c}
\lambda-\lambda_{r} \\
\lambda-\hat{\lambda} \\
\lambda_{r}-\hat{\lambda}_{r}
\end{array}\right)
$$

where $\lambda, \lambda_{r}$ and $\left(\hat{\lambda}, \hat{\lambda}_{r}\right)$ are defined in (4b), (5b) and (11b), respectively.

Consider the Lyapunov function $V(e)=e^{\top}\left[\begin{array}{cc}\alpha P & 0 \\ 0 & \beta \bar{P}\end{array}\right] e$ for some $\alpha, \beta>0$ to be specified later. The derivative of $V$ along the trajectories of the closed-loop system satisfies the following relations: 


$$
\begin{aligned}
\dot{V}(e)= & \alpha e_{x}^{\top}\left(P(A+B K)+(A+B K)^{\top} P\right) e_{x} \\
& +2 \alpha e_{x}^{\top} P B W e_{\xi} \\
& +e_{\xi}^{\top}\left(\beta \bar{P}(\bar{A}-L \bar{C})+(\bar{A}-L \bar{C})^{\top} \beta \bar{P}\right) e_{\xi} \\
& +2 \alpha e_{x}^{\top} P G\left(\lambda-\lambda_{r}\right)+2 \beta e_{\xi}^{\top} \bar{P} \bar{G}\left(\begin{array}{c}
\lambda-\hat{\lambda} \\
\lambda_{r}-\hat{\lambda}_{r}
\end{array}\right) \\
= & -\alpha e_{x}^{\top} Q e_{x}-\beta e_{\xi}^{\top} \bar{Q} e_{\xi}+2 \alpha e_{x}^{\top} P B W e_{\xi} \\
& +2 \alpha e_{x}^{\top} H^{\top}\left(\lambda-\lambda_{r}\right)+2 \beta \tilde{x}^{\top} H^{\top}(\lambda-\hat{\lambda}) \\
& +2 \beta e_{r}^{\top} H_{r}^{\top}\left(\lambda_{r}-\hat{\lambda}_{r}\right) .
\end{aligned}
$$

Using the assumption that $H \Pi=H_{r}$, it now follows from the definition of the normal cone that

$$
\begin{aligned}
e_{x}^{\top} H^{\top}\left(\lambda-\lambda_{r}\right) & =\left\langle H x-H \Pi x_{r}, \lambda-\lambda_{r}\right\rangle \\
& =\left\langle H x-H_{r} x_{r}, \lambda-\lambda_{r}\right\rangle \leq 0 \\
\tilde{x}^{\top} H^{\top}(\lambda-\hat{\lambda}) & =\langle H x-H \hat{x}, \lambda-\hat{\lambda}\rangle \leq 0 \\
e_{r}^{\top} H_{r}^{\top}\left(\lambda_{r}-\hat{\lambda}_{r}\right) & =\left\langle H_{r} x-H_{r} \hat{x}_{r}, \lambda_{r}-\hat{\lambda}_{r}\right\rangle \leq 0 .
\end{aligned}
$$

Plugging these relations in the expression for $\dot{V}$, and using the notation $\sigma_{\min }$ to denote the smallest eigenvalue of a matrix and $\gamma$ to denote the induced Euclidean matrix norm of $P B W$, we get

$$
\begin{aligned}
\dot{V}(e) & \leq-\alpha \sigma_{\min }(Q)\left|e_{x}\right|^{2}-\beta \sigma_{\min }(\bar{Q})\left|e_{\xi}\right|^{2}+2 \alpha \gamma\left|e_{x}\right|\left|e_{\xi}\right| \\
& \leq-\alpha \sigma_{\min }(Q)\left|e_{x}\right|^{2}-\beta \sigma_{\min }(\bar{Q})\left|e_{\xi}\right|^{2}+\left|e_{x}\right|^{2}+\alpha^{2} \gamma^{2}\left|e_{\xi}\right|^{2} \\
& =-\left(\alpha \sigma_{\min }(Q)-1\right)\left|e_{x}\right|^{2}-\left(\beta \sigma_{\min }(\bar{Q})-\alpha^{2} \gamma^{2}\right)\left|e_{\xi}\right|^{2} .
\end{aligned}
$$

Thus, choosing $\alpha, \beta$ in the definition of the Lyapunov function $V$ such that $\alpha \sigma_{\min }(Q)>1$ and $\beta \sigma_{\min }(\bar{Q})>\alpha^{2} \gamma^{2}$, renders $\dot{V}$ negative definite. In particular $e_{x}$ converges to zero, from which it follows that $w=C x+D x_{r}=C(x-$ $\left.\Pi x_{r}\right)=C e_{x}$ converges to zero.

Closed-loop stabilization: The closed-loop stabilization could be shown in exactly the same manner as above. The difference being, the derivative of the Lyapunov function $V$ is calculated along the trajectories of the dynamics of $\left(x, \tilde{x}, \hat{x}_{r}\right)$ given in $(13)$.

\section{APPLICATION: POLYHEDRAL CONSTRAINTS}

In this section, we apply our theoretical results to formulate an output regulation problem with polyhedral constraints. In order to do so, the exosystem generating the reference trajectories is expressed in the form of a linear complementarity system (LCS), that is, we consider:

$$
\begin{gathered}
\dot{x}_{r}(t)=A_{r} x_{r}(t)+G_{r} \lambda_{r} \\
0 \leq \lambda_{r} \perp H_{r} x_{r}(t)+g(t) \geq 0
\end{gathered}
$$

where $g(\cdot):[0, \infty) \rightarrow \mathbb{R}^{d_{s}}$ is a locally absolutely continuous function. It will be assumed that the initial condition of (16) are regular and no discontinuities or impulses are introduced in the state trajectory $x_{r}$, so $(\mathbf{H} 4)$ holds (see Camlibel et al. [2002] for solution notions adopted for such systems). The plant to be controlled is:

$$
\dot{x}(t)=A x(t)+B u(t)+F x_{r}(t) .
$$

Our aim is to find a control input $u$ such that the following two objectives are met:

(O1) the output regulation is achieved with respect to the variable $w$ defined in (6), and
(O2) the state $x$ satisfies the constraint that $y(t):=$ $H x(t)+g(t) \geq 0$, for all $t \geq 0$, where the matrix $H$ satisfies $^{2}$

$$
\operatorname{range}(H)-\mathbb{R}_{+}^{d_{s}}=\mathbb{R}^{d_{s}} .
$$

Thus, in addition to the output regulation, we have a viability problem in Aubin's sense since the state of the plant is constrained to evolve within a predefined polyhedron at all times.

To solve the problem at hand, we reformulate the dynamics of (16) in the form of (5). Towards this end, we recall the following fundamental relation from convex analysis:

$$
0 \leq \lambda \perp H x(t)+g(t) \geq 0 \Leftrightarrow-\lambda \in \partial \psi_{\mathbb{R}_{+}^{d_{s}}}(H x(t)+g(t)),
$$

where $\psi_{S}$ denotes the indicator function of the set $S$, that is, $\psi_{S}(x):=0$ if $x \in S$ and $\psi_{S}(x)=+\infty$ if $x \notin S$. For convex sets, the subdifferential (denoted by $\partial$ ) of the indicator function satisfies $\partial \psi_{S}(x)=\mathcal{N}_{S}(x)$. Now, by letting

it follows that

$$
\mathcal{S}(t):=\left\{z \in \mathbb{R}^{d_{s}} \mid z+g(t) \geq 0\right\},
$$

$$
\partial \psi_{\mathbb{R}_{+}^{d_{s}}}(H x(t)+g(t))=\partial \psi_{\mathcal{S}(t)}(H x(t))=\mathcal{N}(\mathcal{S}(t) ; H x(t)) .
$$

Thus (16) is equivalently written as:

$$
\begin{aligned}
& \dot{x}_{r}(t)=A_{r} x_{r}(t)+G_{r} \lambda_{r} \\
& \lambda_{r} \in-\mathcal{N}\left(\mathcal{S}(t) ; H_{r} x_{r}(t)\right)
\end{aligned}
$$

where $\mathcal{S}(t)$ is defined in $(20)$.

It is easily seen that $\mathcal{S}(t)$ satisfies $(\mathbf{H 1})$ and for each $z \in \mathbb{R}^{n}$,

$$
|d(z, \mathcal{S}(t))-d(z, \mathcal{S}(s))| \leq|g(t)-g(s)|,
$$

that is, (H2) holds. Moreover, each $\mathcal{S}(t)$ intersects nontrivially with the range of $H$ under the condition (18) and thus (H3) holds (see Remark 4).

For the plant under consideration, we split the control input $u$ as $u:=u_{\text {im }}+u_{\lambda}$ with the motivation that $u_{\text {im }}$ refers to the classical internal model control input of the form (9) and $u_{\lambda}$ would play the role of normal vector required to maintain the constraint (a viability controller). Thus, the following control system is realized:

$$
\begin{gathered}
\dot{x}(t)=A x(t)+B u_{\mathrm{im}}(t)+B u_{\lambda}(t)+F x_{r}(t), \\
u_{\lambda}(t) \in-\mathcal{N}(\mathcal{S}(t) ; H x(t)) \\
u_{\mathrm{im}}(t)=K x+(M-K \Pi) x_{r}(t)
\end{gathered}
$$

where $M$ and $\Pi$ are obtained as solutions to (8) with $G$ replaced by the matrix $B$, and $K$ is chosen such that $(A+B K, B, H)$ is strictly passive.

Corollary 9. The system (21) possess a unique solution and the resulting state trajectory $x(\cdot)$ satisfies the properties (O1) and (O2).

It may be the case that equation (21b) is not easily computable, and thus we replace it with an alternate expression in the form of complementarity relation for which there exist efficient solvers. It may be verified that

$\overline{2 \text { Equation (18) means that for each } y} \in \mathbb{R}^{d_{s}}$, there exists $x \in \mathbb{R}^{n}$ and $z \in R_{+}^{d_{s}}$ such that $H x-z=y$. Clearly, (18) holds if $H$ is surjective but may also hold in other cases. 


$$
\begin{gathered}
u_{\lambda}(t) \in-\mathcal{N}(\mathcal{S}(t) ; H x(t)) \\
\Leftrightarrow 0 \leq u_{\lambda}(t) \perp y(t)=H x(t)+g(t) \geq 0 \\
\Leftrightarrow \begin{cases}u_{\lambda}(t)=0 & \text { if } H x(t) \in \operatorname{int} \mathcal{S}(t) \\
0 \leq u_{\lambda}(t) \perp \dot{y}(t) \geq 0 & \text { if } H x(t) \in \text { bd } \mathcal{S}(t)\end{cases}
\end{gathered}
$$

where $\dot{y}=H A x+H B u_{\mathrm{im}}+H B u_{\lambda}+H F x_{r}+\dot{g}$ is considered to be known.

\section{CONCLUSIONS}

This paper studied the problem of output regulation in a certain class of nonsmooth dynamical systems that model state trajectories constrained within some closed, convex, and time-varying set. The classical internal model principle was used to derive conditions to synthesize a control law that achieves the desired objective. The analysis were based on using the Lyapunov methods in combination with monotonicity property of the normal cone operator to prove stability.

Several directions of research could stem from this work. One can easily generalize this approach to consider cases where the variation of the set-valued mapping $\mathcal{S}(\cdot)$ is of locally bounded variation which allows for discontinuities in the states as well, although one has to use certain tools from measure theory to formalize results in this direction as done in Tanwani et al. [2013a,b]. A more interesting problem would be to consider cases where the set-valued mapping $\mathcal{S}(\cdot)$ is not just time-dependent, but also statedependent. This would allow us to model mechanical systems with impacts within our framework but deeper investigation is required for the synthesis of control in such cases. One can also consider more general classes of LCS which allow for modeling of power converters with switching devices (see Vasca et al. [2009]) and the approach adopted in this paper could provide useful methodology for designing switching laws to regulate the output of such systems.

\section{REFERENCES}

J. J. Benjamin Biemond, N. van de Wouw, W. P. M. H. Heemels, and H. Nijmeijer. Tracking control for hybrid systems with state-triggered jumps. IEEE Trans. Automatic Control, 58(4):876 - 890, 2013.

B. Brogliato. Nonsmooth Mechanics. Springer-Verlag London, 2nd edition, 1999.

B. Brogliato and W.P.M.H. Heemels. Observer design for Lur'e systems with multivalued mappings: A passivity approach. IEEE Trans. Automatic Control, 54(8):1996 - 2001, 2009.

C. I. Byrnes and A. Isidori. Internal model principle for linear systems with periodic state jumps. IEEE Trans. Automatic Control, 35(2):131 - 140, 1990.

C. I. Byrnes and A. Isidori. Output regulation for nonlinear systems: An overview. Int. J. Robust \& Nonlinear Control, 10:323 - 337, 2000.

M. K. Camlibel, W. P. M. H. Heemels, and J. M. Schumacher. On linear passive complementarity systems. European J. of Control, 8(3):220 - 237, 2002.

C. De Persis and B. Jayawardhana. On the internal model principle in formation control and in output synchronization of nonlinear systems. In Proc. 51st IEEE Conf. Decision \& Control, pages 4894 - 4899, Maui, Hawaii, December 2012.
J. F. Edmond and L. Thibault. Relaxation of an optimal control problem involving a perturbed sweeping process. Math. Program., Ser. B, 104:347 - 373, 2005.

F. Forni, A. Teel, and L. Zaccarian. Follow the bouncing ball: Global results on tracking and state estimation with impacts. IEEE Trans. Automatic Control, 58(6): $1470-1485,2013$.

B. Francis. A linear multivariable regulator problem. SIAM J. Control and Optimization, 15(3):486 - 505, 1977.

S. Galeani, L. Menini, and A. Potini. Robust trajectory tracking for a class of hybrid systems: An internal model principle approach. IEEE Trans. Automatic Control, 57 (2):344 - 359, 2012.

W. P. M. H. Heemels, M. K. Camlibel, J. M. Schumacher, and B. Brogliato. Observer-based control of linear complementarity systems. Int. J. Robust $\&$ Nonlinear Control, 21(10):1193 - 1218, 2011.

L. Marconi and A. Teel. Internal model principle for linear systems with periodic state jumps. IEEE Trans. Automatic Control, 58(11):2788 - 2802, 2013.

I. C. Morarescu and B. Brogliato. Trajectory tracking control of multiconstraint complementarity Lagrangian systems. IEEE Trans. Automatic Control, 55(6):1300 1313, 2010

J. J. Moreau. Evolution problem associated with a moving convex set in Hilbert space. J. Differential Equations, 26:347 - 374, 1977.

R. T. Rockafellar and R. J-B Wets. Variational Analysis, volume 317 of Gundlehren der mathematischen Wissenchaften. Springer-Verlag, Berlin, 3rd printing, 2009 edition, 1998.

A. Serrani, A. Isidori, and L. Marconi. Semiglobal nonlinear output regulation with adaptive internal model. IEEE Trans. Automatic Control, 46(8):1178 - 1194, 2001.

A. Tanwani and D. Liberzon. Invertibility of switched nonlinear systems. Automatica, 46(12):1962 - 1973, 2010 .

A. Tanwani, B. Brogliato, and C. Prieur. Stability and observer design for a class of nonsmooth dynamical systems with nonconvex state constraints. Research Report RR - 8248, INRIA, Rhône-Alpes, 2013a. Available online: http://hal.inria.fr/hal-00790618.

A. Tanwani, B. Brogliato, and C. Prieur. Passivity-based observer design for a class of Lagrangian systems with perfect unilateral constraints. In Proc. 52nd IEEE Conf. Decision $\&$ Control, Florence, Italy, December 2013b.

F. Vasca, M. K. Camlibel, L. Iannelli, and R. Frasca. A new perspective for modeling power electronics converters: Complementarity framework. IEEE Trans. on Power Electronics, 24(2):456 - 468, 2009.

$\mathrm{L}$. $\mathrm{Vu}$ and $\mathrm{D}$. Liberzon. Invertibility of switched linear systems. Automatica, 44(4):949 - 958, 2008.

P. Wieland, R. Sepulchre, and F. Allgower. An internal model principle is necessary and sufficient for linear output synchronization. Automatica, 47(5):1068 - 1074, 2011. 